\title{
miR-218 is downregulated and directly targets SH3GL1 in childhood medulloblastoma
}

\author{
JIPENG SHI $^{*}$, LIUZHONG YANG $^{2 *}$, TUANJIE WANG $^{3}$, JIAN ZHANG $^{3}$, \\ XIXIA GUO ${ }^{3}$, XIAOQING HUO ${ }^{2}$ and HONGRUI NIU ${ }^{2}$ \\ Departments of ${ }^{1}$ Neonatology, ${ }^{2}$ Medical Oncology and ${ }^{3}$ Pediatrics, \\ The First Affiliated Hospital of Xinxiang Medical University, Weihui, Henan 453100, P.R. China
}

Received March 5, 2013; Accepted August 8, 2013

DOI: $10.3892 / \mathrm{mmr} .2013 .1639$

\begin{abstract}
An increasing number of studies have suggested that microRNAs (miRNAs) are aberrantly expressed in numerous types of tumors and that a deregulation in miRNA expression may lead to carcinogenesis. Although miR-218 has been demonstrated to be downregulated in several types of cancer, including medulloblastoma (MB), its involvement in $\mathrm{MB}$ is unclear. In the present study, the expression of miR-218 and SH3GL1 were assessed in four MB cell lines and normal cerebellum by qPCR. The ectopic expression of miR-218 induced by lentiviral transfection in MB cells on proliferation was evaluated by MTT assay, and cell migration and invasion were determined by transwell assays. Analysis of the target protein expression and related protein expression was determined by western blot analysis. The targeting of SH3GL1 by miR-218 was identified using a luciferase reporter assay. The results demonstrated that miR-218 was significantly downregulated in MB cell lines. MiR-218 significantly inhibited SH3GL1 mRNA and protein expression and reduced the luciferase activity of a SH3GL1 3' untranslated region-based reporter. Furthermore, overexpression of miR-218 induced by transfection with lentivirus significantly suppressed $\mathrm{MB}$ cell growth, migration and invasion in vitro. Small interfering RNA-mediated SH3GL1 downregulation partially phenocopied the effect of miR-218 overexpression in the MB cell lines. The results indicated that miR-218 was significantly downregulated in MB cancer cell lines. Furthermore, miR-218 functioned as a tumor
\end{abstract}

Correspondence to: Professor Hongrui Niu, Department of Medical Oncology, The First Affiliated Hospital of Xinxiang Medical University, 88 Jiankang Road, Weihui, Henan 453100, P.R. China

E-mail: hongruiniu@126.com

*Contributed equally

Key words: microRNA, miR-218, targets, medulloblastoma, SH3GL1, tumor suppressor suppressor by regulating SH3GL1 expression in MB cancer cells.

\section{Introduction}

Medulloblastoma (MB) is the most common type of malignant brain tumor in children and is characterized by frequent proliferation and metastasis (1). Successful treatments, including surgery, radiation and chemotherapy, have improved in recent years; however, these therapies result in side effects, including endocrinopathies, impaired cognition and vasculopathies $(2,3)$, and survival rates remain to be improved. Thus, the elucidation of the molecular mechanisms that drive cell proliferation and invasion may provide more effective therapeutic strategies.

MicroRNAs (miRNAs) are an abundant group of endogenous, small, non-coding RNAs that regulate gene expression at the post-transcriptional level; miRNAs undergo base pairing with target mRNAs in the 3' untranslated region (3'UTR), leading to translational inhibition and/or mRNA degradation (4-7). Numerous studies have demonstrated significantly altered miRNA expression patterns in types of human cancer, deregulation of miRNA expression and the contribution of miRNAs to the multistep processes of carcinogenesis either as oncogenes or as tumor-suppressor genes $(8,9)$. A number of miRNA expression profiling studies have demonstrated that miRNA expression is dysregulated, by the comparison of MBs with normal cerebellum (10-12). A previous study determined that the expression of miR-17 92 was upregulated in MBs and promoted cell proliferation (13). Elevated miR-21 expression has been causally linked with cellular mobility and migration of $\mathrm{MB}$ in vitro due to the suppression of the PDCD4 tumor suppressor (14). The expression of miR-218 was identified to be significantly decreased in MB compared with that of normal cerebellum, and miR-218 negatively regulated the CDK6, Rictor and CTSB proto-oncogenes (15). However, the molecular mechanism of miR-218 in MB remains unclear.

The present study aimed to observe the expression of miR-218 in MB cell lines and in vitro experiments were performed to determine the effect of miR-218 on cell growth and invasion. Using reporter assays, miR-218 was identified to directly target the 3'UTR of SH3GL1, which may aid in the elucidation of a potential molecular therapeutic target for human MB cell lines. 


\section{Materials and methods}

Cell culture. Daoy, D458 and PFSK human MB cell lines were purchased from the American Type Culture Collection (Manassas, VA, USA). UW228 and HEK-293T cells were provided by Professor Wu (University of Fudan, Shanghai, China). All cell lines were cultured in Dulbecco's Modifed Eagle's Medium (Gibco-BRL, Grand Island, NY, USA) supplemented with $10 \%$ fetal bovine serum (Gibco-BRL), penicillin $(10 \mathrm{IU} / \mathrm{ml})$ and streptomycin $(10 \mu \mathrm{g} / \mathrm{ml})$. All cells were maintained in humidified air at $37^{\circ} \mathrm{C}$ and $5 \% \mathrm{CO}_{2}$. Cells were passaged a maximum of eight times.

RNA extraction and qPCR analyses. Total RNA was isolated from cells with TRIzol reagent (Invitrogen Life Technologies, Carlsbad, CA, USA) and treated with DNA-free kit (Invitrogen Life Technologies) to remove any remaining DNA, according to the manufacturer's instructions. qPCR assays were performed in triplicate to detect the miR-218 and SH3GL1 expression using the PrimeScript RT reagent kit (Takara Bio, Inc., Shiga, Japan) and the SYBR Green PCR Master Mix kit (Qiagen, Hilden, Germany) according to the manufacturer's instructions, with the ABI PRISM ${ }^{\circledR} 7900$ HT Sequence Detection system (Invitrogen Life Technologies).

For miRNA detection, gene specific primers were utilized. The U6 small nuclear RNA was used as a control to determine the relative miRNA expression. The RT primers were designed as follows: 5'-GTCGTATCCAGTGCAGGGTCCGAGG TATTCGCACTGGATACGACACATGG-3' for miR-218; 5'-AACGCTTCACGAATTTGCGT-3' for U6. The PCR primers for mature miR-218 and U6 were designed as follows: Sense: 5'-CGGGCTTGTGCTTGATCTA-3' and antisense: 5'-GTGCAGGGTCCGAGGT-3' for miR-218; sense: 5'-CTCGCTTCGGCAGCACA-3' and antisense: 5'-AACGCTTCACGAATTTGCGT-3' for U6.

For the analysis of the SH3GL1 mRNA expression, $500 \mathrm{ng}$ aliquots of total RNA were used to synthesize cDNA at a final volume of $10 \mu 1$, and glyceraldehyde 3-phosphate dehydrogenase (GAPDH) was used as the internal control. The primer sequences were as follows: Sense: 5'-ATCGTCTTTCCGATCTTCCG-3' and antisense: 5'-TGCCCTCGTACCAGTTCTCAT-3' for SH3GL1; sense: 5'-GGGAGCCAAAAGGGTCAT-3' and antisense: 5'-GAGTCCTTCCACGATACCAA-3' for GADPH. The PCR cycle settings were as recommended by Qiagen: $95^{\circ} \mathrm{C}$ for $10 \mathrm{~min} ; 40$ amplification cycles at $95^{\circ} \mathrm{C}$ for $10 \mathrm{sec}$ and $60^{\circ} \mathrm{C}$ for $1 \mathrm{~min}$.

Plasmid construction and transfection. To construct a luciferase reporter vector, a cDNA fragment encoding the SH3GL 3'UTR from UW228 cells was amplified by PCR and cloned downstream of the Renilla luciferase gene in psicheck2 (Promega Corporation, Madison, WI, USA). The primer sequences used were as follows: Sense: 5'-AAAGTTTAAACATCGTCTTTC CGATCTTCCG-3' and antisense: 5'-AAAGCGGCCGCTGC CCTCGTACCAGTTCTCAT-3' for SH3GL1. Psicheck2 luciferase constructs containing mutant 3'UTR (AAGCACAA to AACGAGAA) were also generated using the QuikChange site-directed mutagenesis kit (Stratagene, La Jolla, CA, USA) and the vectors were termed SH3GL1-UTR-WT and
SH3GL1-UTR-MUT, respectively. Positive clones were identified by PCR screening and DNA sequencing.

Knockdown experiments were performed by the transient transfection of SH3GL1 siRNA using Lipofectamine ${ }^{\mathrm{TM}} 2000$ (Invitrogen Life Technologies) according to the manufacturer's instructions. The cell lysates were harvested $48 \mathrm{~h}$ following transfection for western blot analysis. The sequence of the SH3GL1 siRNA was obtained from Origene (SR304356; Rockville, MD, USA). Briefly, for the reporter assays, cells were transiently cotransfected with wild-type or mutant reporter plasmids and miR-218. Renilla and Firefly luciferase activities were measured $36 \mathrm{~h}$ following transfection using the Dual-Luciferase assay (Promega Corporation) and the results were normalized with Firefly luciferase. Each reporter plasmid was transfected at least three times (on different days) and samples were assayed in triplicate.

Lentivirus packaging, infection and stable cell generation. The lentiviral vector (pCDH-Vector) was obtained from System Biosciences (Mountain View, CA, USA). A total of $16 \mu \mathrm{g}$ of the plasmids, including pCDH-miR-218 or pCDH-Vector, TAT, GAG, REC and VSVG, were cotransfected with $40 \mu \mathrm{l}$ Lipofectamine 2000 (Invitrogen Life Technologies) into HEK-293T cells in a $100-\mathrm{mm}$ diameter culture dish. The supernatant was collected $60 \mathrm{~h}$ following infection, filtered through a $0.45-\mu \mathrm{m}$ pore filter and used as the source of the virus. PFSK and UW228 cells were infected with either pCDH-Vector or pCDH-Vector with $8 \mu \mathrm{g} / \mathrm{ml}$ polybrene (Sigma-Aldrich, St. Louis, MO, USA) for $24 \mathrm{~h}$, and the medium was replaced. The efficiency of infection was measured under a fluorescent microscope $72 \mathrm{~h}$ following infection.

Cell proliferation. Cells were plated at a density of 4,000 cells/well, in 4 wells of 96 -well plates containing complete medium. Cell proliferation was detected using CellTiter 96 AQueous One Solution (Promega Corporation) at $0,24,48$ and $72 \mathrm{~h}$ following plating. For measurement of cell proliferation, $20 \mu \mathrm{l}$ methanethiosulfonate reagent was added to the medium and incubated at $37^{\circ} \mathrm{C}$ in a humidified $5 \% \mathrm{CO}_{2}$ atmosphere for $1 \mathrm{~h}$. The absorbance was read at $490 \mathrm{~nm}$ using a 96-well microplate reader (ELx800 Absorbance Microplate Reader; BioTek, Winooski, VT, USA).

Invasion assays. The invasive ability of the cells was determined using 24-well Matrigel Invasion Chambers according to the manufacturer's instructions (Corning Life Sciences, Corning, NY, USA). Media (600 $\mu$ l) containing 10\% FBS was added to the lower chamber and a cell suspension of $2 \times 10^{5}$ cells in $100 \mu 1$ DMEM medium was added into each well of the top chamber. Subsequent to this, the cells were incubated for $48 \mathrm{~h}$ at $37^{\circ} \mathrm{C}$ in a humidified incubator with $5 \% \mathrm{CO}_{2}$. Cells that had passed through the membrane were stained with methanol and $0.1 \%$ crystal violet, imaged and counted using an IX71 inverted microscope (Olympus, Tokyo, Japan) from three random microscope fields per filter. Experiments were repeated three times.

Western blot analysis. Cells were washed with phosphate-buffered saline and lysed with RIPA lysis buffer (CST) supplied with protease and phosphatase inhibitor cocktails 
A

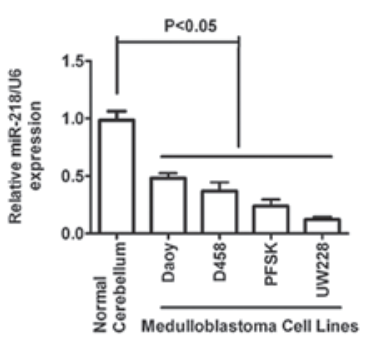

B

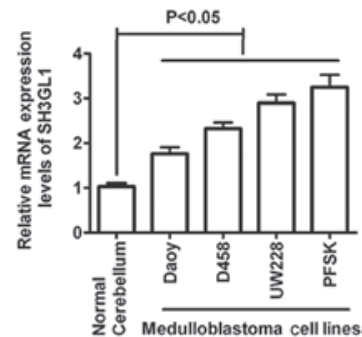

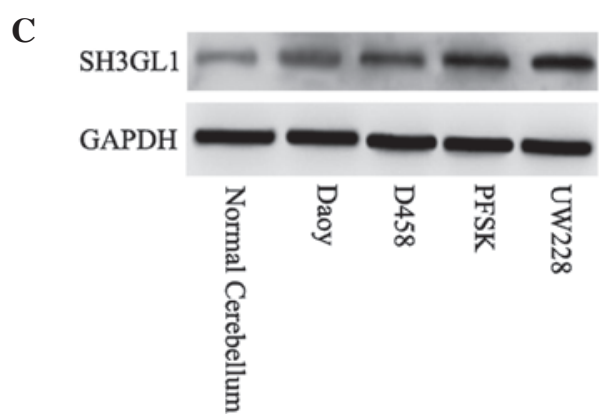

Figure 1. miR-218 expression is downregulated and inversely correlated with the expression of SH3GL1 in MB cancer cell lines. (A) qPCR analysis of the miR-218 expression levels in a panel of human MB cells and in normal cerebellum. (B and C) SH3GL1 expression at the mRNA and protein levels in different human MB cell lines and normal cerebellum. MB, medulloblastoma; GAPDH, glyceraldehyde 3-phosphate dehydrogenase.

(1\%; Sigma-Aldrich). Total denatured proteins (50 $\mu \mathrm{g})$ were subjected to sodium dodecyl sulfate (SDS)-polyacrylamide gel electrophoresis (10\% SDS-acrylamide gel) and transferred onto polyvinylidene fluoride membranes (Bio-Rad, Hercules, CA, USA). The membrane was incubated with SH3GL1 (TA309473, Origene), Phospho-c-Jun (Ser73; \#9164; Cell Signaling Technology, Inc., Beverly, MA, USA), Phospho-p44/42 MAPK [extracellular signal-regulated kinases (Erk)1/2; Thr202/Tyr204; \#9101; Cell Signaling Technology, Inc.] and GAPDH antibodies (10494-1-AP, Proteintech $^{\mathrm{TM}}$, Chicago, IL, USA). Primary antibodies were detected with horseradish peroxidase-conjugated secondary antibodies (Santa Cruz Biotechnology, Inc., Santa Cruz, CA, USA) and the membranes were subjected to a chemiluminescence detection assay.

Statistical analysis. All experiments were performed at least in triplicate. Values are presented as the mean \pm standard deviation. Significance was examined by Student's t-test (two-tailed) or one-way analysis of variance. $\mathrm{P}<0.05$ was considered to indicate a statistically significant difference.

\section{Results}

miR-218 is downregulated in $M B$ and its expression is inversely correlated with that of SH3GL1. To investigate the involvement of miR-218 in MB, the miR-218 expression level was detected using qPCR in a number of selected cancer cell lines as well as in normal cerebellum (Fig. 1A). The expression level of miR-218 was significantly lower or undetectable in all tested cancer cell lines compared with that of the normal cerebellum. To identify the proteins that miR-218 affected, the mRNA targets were predicted by bioinformatics. Among the list of potential targets, the seed sequence of miR-218 was complementary to the 3'-UTR of SH3GL1. To investigate the correlation between the expression of miR-218 and SH3GL1, the expression of SH3GL1 at the mRNA and protein levels were determined in the same panel of cell lines. As expected, the mRNA and protein expression levels of SH3GL1 were higher in the cancer cell lines when compared with that in the normal cerebellum (Fig. 1B and C). The results indicated that a potential tumor suppressor role of miR-218 is downregulated in MB, which may have directly targeted SH3GL1.

miR-218 directly targets the 3'UTR of SH3GL1. To determine whether miR-218 regulates SH3GL1, a reporter plasmid (in which the full-length 3'-UTR of SH3GL1 was inserted downstream of the Renilla luciferase gene) was generated for reporter gene assays (Fig. 2A). The transient transfection of HEK293T cells with the SH3GL1-UTR-WT or SH3GL1-UTR-MUT construct and miR-218 resulted in a significant reduction in reporter gene expression when compared with that of the control vector (Fig. 2B). Mutations in the targeting sites of miR-218 were unaffected by the simultaneous transfection with miR-218 (Fig. 2B). To further confirm that miR-218-mediated reduction of the luciferase activity of SH3GL1 was due to the direct interaction between miR-218 and its putative binding site, cotransfection of SH3GL1-UTR-WT or SH3GL1-UTR-MUT with anti-miR-218 in HEK293T cells was conducted. As expected, anti-miR-218 markedly increased the luciferase activity of SH3GL1-UTR-WT compared with that of anti-miR-normal cerebellum (NC); however, the effect was completely abolished in this mutant construct (Fig. 2C).

Significance of SH3GL1 suppression. To further address the significance of the suppression of SH3GL1 by miR-218 in MB cells, PSFK and UW228 cells stably expressing miR-218 were generated and the overexpression of miR-218 was confirmed by qPCR analysis (Fig. 2D). The mRNA and protein levels of SH3GL1 by qPCR and western blot analysis demonstrated that the overexpression of miR-218 downregulated the endogenous SH3GL1 levels (Fig. 2E and F). These 
A

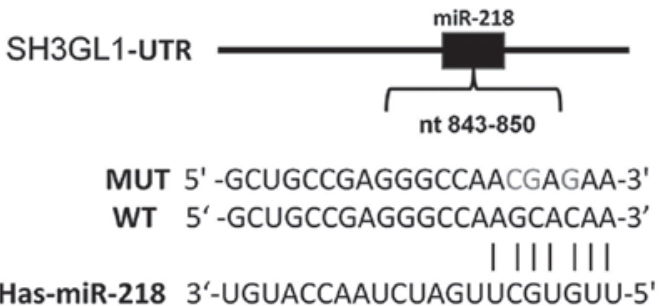

B

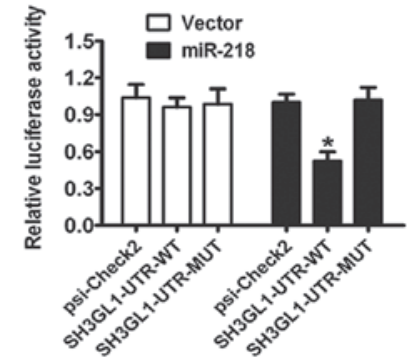

D

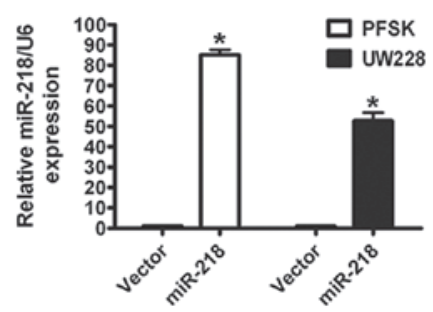

C

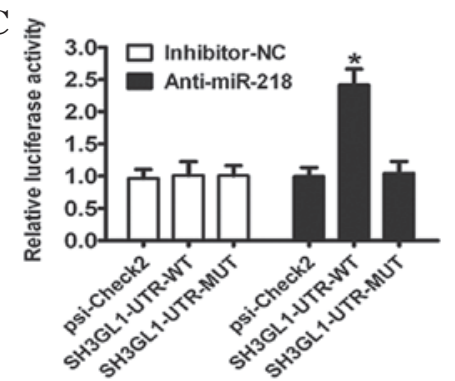

$\mathbf{E}$

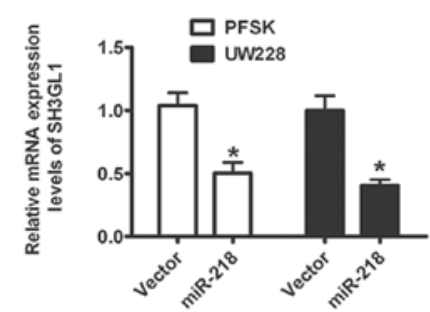

$\mathbf{F}$

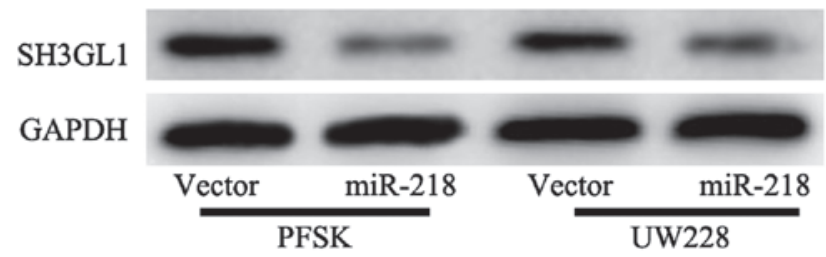

Figure 2. SH3GL1 is a target of miR-218. (A) Predicted miR-218 target sequences in the 3'-UTR of SH3GL1 (SH3GL1-UTR-WT) and mutants containing three mutated nucleotides in the 3'-UTR of SH3GL1 (SH3GL1-UTR-MUT). (B and C) HEK-293T cells were cotransfected with miR-218 or anti-miR-218 and psicheck2 vector with SH3GL1 3'-UTR, WT or mutated. After $36 \mathrm{~h}$, the luciferase activity was measured. Renilla luciferase activity was normalized to firefly luciferase expression for each sample. Each experiment was performed in triplicate. ${ }^{*} \mathrm{P}<0.05$. (D) Relative miR-218 expression levels in PSFK and UW228 cells stably expressing miR-218 by qPCR. (E and F) qPCR and western blot analysis were performed to detect the expression of SH3GL1 mRNA and protein in PSFK and UW228 cells stably expressing miR-218. UTR, untranslated region; MUT, mutated; WT, wild type; NC, normal cerebellum.

results suggested that $\mathrm{miR}-218$ downregulated the endogenous SH3GL1 expression by directly binding to the 3'-UTR sequence of SH3GL1.

Stable overexpression of $m i R-218$ inhibits cell proliferation and invasion. To assess the biological role of miR-218, the effect of the overexpression of miR-218 on cell proliferation and invasion was determined. MTT assays demonstrated that the overexpression of miR-218 markedly impaired the growth rate of $\mathrm{MB}$ cells as compared with that of the control vector (Fig. 3A and B). Similarly, the results of the invasion assays revealed that invasive ability was decreased following the overexpression of miR-218 in PSFK and UW228 cells (Fig. 3C and D). In conclusion, these results demonstrated that miR-218 inhibited MB cell proliferation and invasion.

miR-218 suppresses SH3GL1 and negatively regulates the ERK pathway in MB cell lines. To confirm the involvement of
SH3GL1 in the miR-218-mediated inhibition of cell growth, siRNA targeting SH3GL1 was used to downregulate SH3GL1 expression and analyze its effect on cell growth. The protein expression levels of SH3GL1, phosphorylated (p)-ERK and p-Jun were significantly downregulated in PSFK and UW228 cells with siSH3GL1 compared with those of the normal cerebellum (Fig. 4A and B). PSFK and UW228 cells in which SH3GL1 had been knocked down demonstrated a decrease in growth rate in the MTT assay (Fig. 4C and D), which was similar to the phenotype observed following miR-218 overexpression in PSFK and UW228 cells. The expression of p-ERK following ectopic expression of miR-218 in MB cells was also observed. Consistent with the effect of SH3GL1, miR-218 overexpression significantly reduced the expression of p-ERK and p-Jun in PSFK and UW228 cells and induced the maintained overexpression of miR-218 (Fig. 4A and B). In conclusion, the tumor-suppressive role of miR-218 may mediate SH3GL1 via the mitogen-activated protein kinase pathway. 

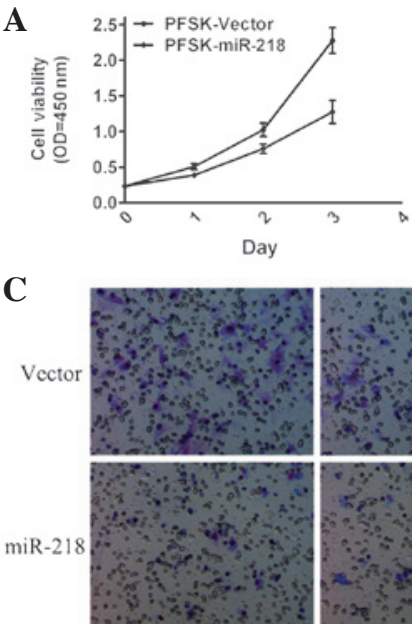

PFSK

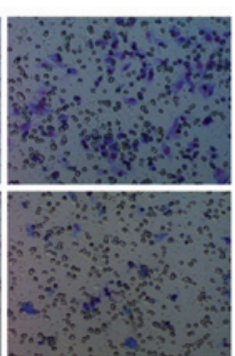

UW228

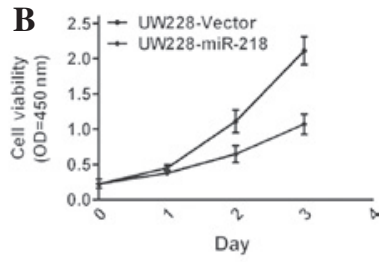

D

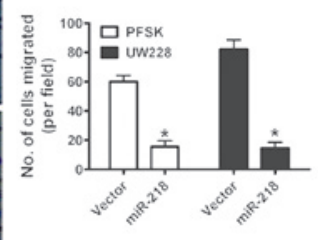

Figure 3. Effect of miR-218 on the cell proliferation and invasion of MB cells. (A and B) Cell proliferation of PSFK or UW228 cells with or without miR-218 overexpression were determined by an MTT assay. Data are presented as the mean \pm SD from three independent experiments. (C and D) Invasion assays were used to investigate the invasive ability of $\mathrm{MB}$ cells by transwell assays. ${ }^{*} \mathrm{P}<0.05$. MB, medulloblastoma.

A

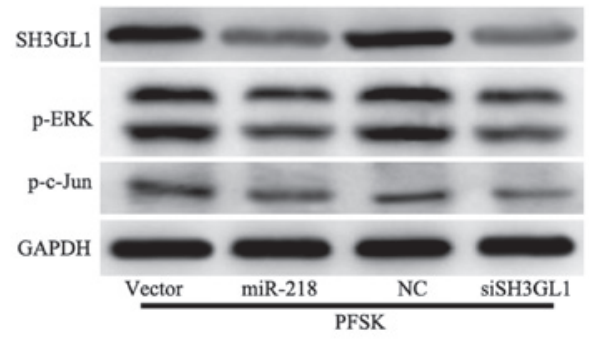

B

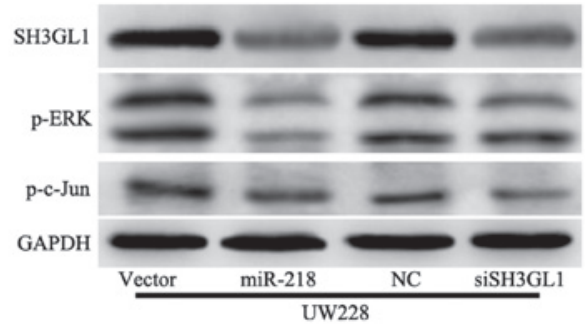

C

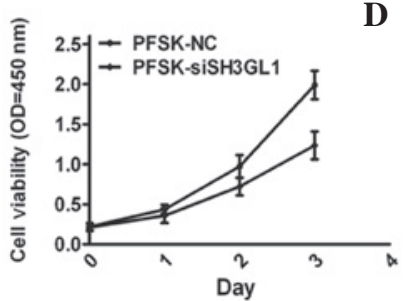

D

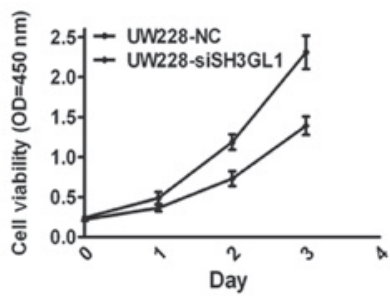

Figure 4. SH3GL1 knockdown phenocopies the miR-218 overexpression in MB cells. (A and B) Expression of SH3GL1, p-ERK and p-Jun were detected by western blot analysis in PSFK or UW228 cells following miR-218 overexpression and transfection with siSH3GL1. (C and D) Cell proliferation analysis of transfected PSFK or UW228 cells with siSH3GL1 or NC by MTT assay at 24, 48 and $72 \mathrm{~h}$ following transfection. MB, medulloblastoma; NC, normal cerebellum; GAPDH, glyceraldehyde 3-phosphate dehydrogenase; p-ERK, phosphorylated-extracellular signal-regulated kinases.

\section{Discussion}

Understanding of the biological mechanisms of malignant brain tumors, such as MB, is essential for advances in therapy $(13,16-18)$. Numerous studies observed the aberrant expression of microRNAs in MB and suggested that the determination of key microRNAs may lead to the identification of novel targets for more effective therapies with fewer side effects $(14,15)$. The reduced expression of miR-218 was also demonstrated in gastric, colon, prostate, pancreatic, glioma cell and cervical cancer (19-21). In the present study, low expression levels of miR-218 were identified in MB cell lines compared with in normal cerebellum; the expression of miR-218 was significantly downregulated in all examined 
MB cell lines, including Daoy, D458, PFSK and UW228, compared with in normal cerebellum. This was consistent with the previous studies, which demonstrated that miR-218 functions as a tumor suppressor in MB (15).

Identification of putative miRNA targets is important for the understanding of the function of miRNAs. In this study, we identified SH3GL1 as a direct target of miR-218 with the aid of bioinformatics, and demonstrated that the upregulation of miR-218 markedly reduced the endogenous SH3GL1 expression at the transcriptional and protein levels in MB cells. SH3GL1 has been observed to be involved in endocytosis and signal transduction $(22,23)$. The epidermal growth factor receptor (EGFR) signaling pathway is an important pathway that regulates cellular proliferation, differentiation and invasion (24). SH3GL1 binds to BPGAP1, which is involved in the activation of EGFR endocytosis and ERK1/2 signaling. In addition, overexpression of SH3GL1 has been demonstrated to promote cell growth $(25,26)$.

The reduced expression of miR-218 in MB suggested that miR-218 acted as a tumor suppressor. The overexpression of miR-218 suppressed cell growth and invasion in vitro. It has been demonstrated that miR-218 inhibited the growth of oral cancer by targeting the mTOR component Rictor with the inhibition of Akt phosphorylation (27). Exogenous expression of miR-218 suppressed the growth of nasopharyngeal carcinoma by BIRC5 and suppressed cell invasion via the SLIT-ROBO pathway (28). Previously, miR-218 was observed to be downregulated in $\mathrm{MB}$ and the restoration of miR-218 resulted in a marked decrease in MB cell growth, colony formation, migration and invasion, as well as tumor sphere size, by directly targeting CDK6, RICTOR and CTSB (15). However, miR-218 has been determined to stimulate the Wnt pathway by downregulating SOST, DKK2 and SFRP2 during the process of osteogenesis (29), which is inconsistent with our findings. This inconsistency may be due to the use of different cancer cell lines (gastric and lung), and therefore the organ-specific targets and cellular context may differ.

To further determine the effect of miR-218 on MB, SH3GL1 was knocked down in PFSK and UW228 cells, and the proliferation was decreased, which was similar to the phenotype observed following miR-218 overexpression in PFSK and UW228 cells. Previous studies implicated that SH3GL1 activated P-ERK activity, which regulates cancer cell growth and migration. In the current study, overexpression of miR-218 decreased the expression of P-ERK and P-Jun, which was consistent with the results of the knockdown of SH3GL1. Thus, this study demonstrated that miR-218 exerted tumor suppressor effects in MB cells by downregulating SH3GL1. The mechanisms underlying SH3GL1 in carcinogenesis require further investigation.

In conclusion, the present study confirmed that miR-218 is a tumor suppressor miRNA involved in the initiation and progression of human MB, by affecting multiple signal pathways. These results also suggested that miR-218 may provide therapeutic potential for the treatment of MB.

\section{Acknowledgements}

This study was supported by grants from the National Natural Science Foundation of China (grant no. 81201765).

\section{References}

1. Gilbertson RJ and Ellison DW: The origins of medulloblastoma subtypes. Annu Rev Pathol 3: 341-365, 2008.

2. Ellison DW, Kocak M, Dalton J, et al: Definition of disease-risk stratification groups in childhood medulloblastoma using combined clinical, pathologic, and molecular variables. J Clin Oncol 29: 1400-1407, 2011.

3. No authors listed: 43rd Congress of the International Society of Paediatric Oncology (SIOP) 2011, Auckland, New Zealand, 28th-30th October, 2011. SIOP Abstracts. Pediatr Blood Cancer 57: 705-897, 2011.

4. Bartel DP: MicroRNAs: genomics, biogenesis, mechanism, and function. Cell 116: 281-297, 2004.

5. Wu L, Fan J and Belasco JG: MicroRNAs direct rapid deadenylation of mRNA. Proc Natl Acad Sci USA 103: 4034-4039, 2006.

6. Krol J, Loedige I and Filipowicz W: The widespread regulation of microRNA biogenesis, function and decay. Nat Rev Genet 11: 597-610, 2010.

7. Filipowicz W, Bhattacharyya SN and Sonenberg N: Mechanisms of post-transcriptional regulation by microRNAs: are the answers in sight? Nat Rev Genet 9: 102-114, 2008.

8. Shenouda SK and Alahari SK: MicroRNA function in cancer: oncogene or a tumor suppressor? Cancer Metastasis Rev 28: 369-378, 2009.

9. Garzon R, Calin GA and Croce CM: MicroRNAs in cancer. Annu Rev Med 60: 167-179, 2009.

10. Uziel T, Karginov FV, Xie S, et al: The miR-17 92 cluster collaborates with the Sonic Hedgehog pathway in medulloblastoma. Proc Natl Acad Sci USA 106: 2812-2817, 2009.

11. Ferretti E, De Smaele E, Po A, et al: MicroRNA profiling in human medulloblastoma. Int J Cancer 124: 568-577, 2009.

12. Genovesi LA, Anderson D, Carter KW, et al: Identification of suitable endogenous control genes for microRNA expression profiling of childhood medulloblastoma and human neural stem cells. BMC Res Notes 5: 507, 2012.

13. Northcott PA, Fernandez-L A, Hagan JP, et al: The miR-17/92 polycistron is up-regulated in sonic hedgehog-driven medulloblastomas and induced by $\mathrm{N}$-myc in sonic hedgehog-treated cerebellar neural precursors. Cancer Res 69: 3249-3255, 2009.

14. Grunder E, D'Ambrosio R, Fiaschetti G, et al: MicroRNA-21 suppression impedes medulloblastoma cell migration. Eur J Cancer 47: 2479-2490, 2011.

15. Venkataraman S, Birks DK, Balakrishnan I, et al: MicroRNA 218 acts as a tumor suppressor by targeting multiple cancer phenotype-associated genes in medulloblastoma. J Biol Chem 288: 1918-1928, 2013.

16. Shapiro RH and Chang AL: Urgent radiotherapy is effective in the treatment of metastatic medulloblastoma causing symptomatic brainstem edema. Pediatr Blood Cancer 57: 1077-1080, 2011.

17. Gajjar A and Pizer B: Role of high-dose chemotherapy for recurrent medulloblastoma and other CNS primitive neuroectodermal tumors. Pediatr Blood Cancer 54: 649-651, 2010.

18. Taniguchi E, Cho MJ, Arenkiel BR, et al: Bortezomib reverses a post-translational mechanism of tumorigenesis for patched1 haploinsufficiency in medulloblastoma. Pediatr Blood Cancer 53: 136-144, 2009.

19. Volinia S, Calin GA, Liu CG, et al: A microRNA expression signature of human solid tumors defines cancer gene targets. Proc Natl Acad Sci USA 103: 2257-2261, 2006.

20. Song L, Huang Q, Chen K, et al: miR-218 inhibits the invasive ability of glioma cells by direct downregulation of IKK- $\beta$. Biochem Biophys Res Commun 402: 135-140, 2010.

21. Volinia S, Calin GA, Liu CG, et al: A microRNA expression signature of human solid tumors defines cancer gene targets. Proc Natl Acad Sci USA 103: 2257-2261, 2006.

22. Ringstad N, Nemoto $Y$ and De Camilli P: The $\mathrm{SH} 3 \mathrm{p} 4 / \mathrm{Sh} 3 \mathrm{p} 8 / \mathrm{SH} 3 \mathrm{p} 13$ protein family: binding partners for synaptojanin and dynamin via a Grb2-like Src homology 3 domain. Proc Natl Acad Sci USA 94: 8569-8574, 1997.

23. So CW, So CK, Cheung N, et al: The interaction between EEN and Abi-1, two MLL fusion partners, and synaptojanin and dynamin: implications for leukaemogenesis. Leukemia 14: 594-601, 2000.

24. Tanaka K, Babic I, Nathanson D, et al: Oncogenic EGFR signaling activates an mTORC2-NF-kappaB pathway that promotes chemotherapy resistance. Cancer Discov 1: 524-538, 2011. 
25. Lua BL and Low BC: Activation of EGF receptor endocytosis and ERK1/2 signaling by BPGAP1 requires direct interaction with EEN/endophilin II and a functional RhoGAP domain. J Cell Sci 118: 2707-2721, 2005.

26. Ma LH, Liu H, Xiong H, et al: Aberrant transcriptional regulation of the MLL fusion partner EEN by AML1-ETO and its implication in leukemogenesis. Blood 109: 769-777, 2007.

27. Uesugi A, Kozaki K, Tsuruta T, et al: The tumor suppressive microRNA miR-218 targets the mTOR component Rictor and inhibits AKT phosphorylation in oral cancer. Cancer Res 71: 5765-5778, 2011.
28. Fish JE, Wythe JD, Xiao T, et al: A Slit/miR-218/Robo regulatory loop is required during heart tube formation in zebrafish. Development 138: 1409-1419, 2011.

29. Hassan MQ, Maeda Y, Taipaleenmaki H, et al: miR-218 directs a Wnt signaling circuit to promote differentiation of osteoblasts and osteomimicry of metastatic cancer cells. J Biol Chem 287: 42084-42092, 2012. 